\title{
CAUCHY'S FUNCTIONAL EQUATION IN RESTRICTED COMPLEX DOMAINS
}

\author{
WATCHARAPON PIMSERT, VICHIAN LAOHAKOSOL, AND SAJEE PIANSKOOL
}

Received 15 September 2004; Revised 22 March 2006; Accepted 4 April 2006

Using a method modified from that used by Pisot and Schoenberg in 1964-1965, a Cauchy's functional equation with restricted domains in the complex field is solved for uniformly continuous solutions.

Copyright (c) 2006 Hindawi Publishing Corporation. All rights reserved.

\section{Introduction}

Consider the Cauchy's functional equation, hereby called CFE,

$$
f\left(\sum_{j=1}^{A} u_{j} \alpha_{j}\right)=\sum_{j=1}^{A} f\left(u_{j} \alpha_{j}\right) .
$$

Under various assumptions on $A$ and $\alpha_{j}^{\prime} s$, Pisot and Schoenberg [8] solved for monotone solutions of (1.1). In a subsequent paper [9], they treated the case where the domain of solutions is a subset of $\mathbb{R}^{n}$ with the following main result. Let $\alpha_{1}, \alpha_{2}, \ldots, \alpha_{A}$ be elements of $\mathbb{R}^{n}(n<A)$ satisfying the following conditions:

(1) every set of $n$ elements among the $\alpha_{i}$ is linearly independent over $\mathbb{R}$,

(2) the elements $\alpha_{1}, \alpha_{2}, \ldots, \alpha_{A}$ are rationally independent, that is, linearly independent over $\mathbb{Q}$.

Let $S=\left\{\sum_{j=1}^{A} u_{j} \alpha_{j} \mid u_{j} \in \mathbb{N}_{0}\right.$, the set of nonnegative integers $\}, B$ a Banach space and $f$ : $S \rightarrow B$. If $f$ is a uniformly continuous solution of the CFE (1.1), then $f(x)$ admits a unique representation of the form $f(x)=\lambda(x)+\sum_{m=1}^{A} \varphi_{m}(x)$, where $\lambda$ is a linear function from $\mathbb{R}^{n}$ into $B$, and each $\varphi_{m}(m=1,2, \ldots, A)$ is a function from $R_{m}=\left\{u_{m} \alpha_{m}+\sum_{j=1, j \neq m}^{A} k_{j} \alpha_{j} \mid\right.$ $\left.u_{m} \in \mathbb{N}_{0}, k_{j} \in \mathbb{Z}\right\}$ into $B$ satisfying

(1) $\varphi_{m}(0)=0$,

(2) $\varphi_{m}\left(x+\alpha_{j}\right)=\varphi_{m}(x)\left(j \neq m, x \in R_{m}\right)$,

(3) $\varphi_{m}$ is a uniformly continuous function on $R_{m}$ into $B$.

Studying both of Pisot's and Schoenberg's works, we observe two significant features, first, that their method of proof in [9], both beautiful and powerful, can be extended to 
wider classes of functional equations and second, that the rational independence can be replaced by denseness, even though the two conditions are not equivalent. We illustrate these observations by solving similar, but different, CFEs in the complex field together with relevant examples and several remarks relating them with the classical complex CFE (see [1]) and in particular with an old theorem of Erdös about monotone additive functions (see [4]). Our main results say roughly that solutions of complex CFEs, uniformly continuous over some dense subsets and additive with respect to certain real or complex base elements, consist generally of two parts, one linear and the other periodic, and the periodic part disappears if each of its elements has a natural dense subdomain.

\section{Case of real base elements}

In this section, we solve the CFE (1.1) with real base elements $\alpha_{j}$ and Gaussian integer coefficients.

Theorem 2.1. Let $A \in \mathbb{N}, A \geq 2$, and $\alpha_{1}, \alpha_{2}, \ldots, \alpha_{A} \in \mathbb{R}$ be such that the set

$$
S^{+}=\left\{\sum_{m=1}^{A}\left(u_{m}+i v_{m}\right) \alpha_{m} \mid u_{m}, v_{m} \in \mathbb{N}_{0}\right\}
$$

is dense in $\mathbb{C}$. If $f: S^{+} \rightarrow \mathbb{C}$ is a uniformly continuous solution of the functional equation

$$
f\left(\sum_{m=1}^{A}\left(u_{m}+i v_{m}\right) \alpha_{m}\right)=\sum_{m=1}^{A}\left\{f\left(u_{m} \alpha_{m}\right)+f\left(i v_{m} \alpha_{m}\right)\right\}
$$

then $f$ can be uniquely written as

$$
f(x)=\lambda(x)+\sum_{m=1}^{A} \varphi_{m}(x)
$$

where $\lambda: \mathbb{C} \rightarrow \mathbb{C}$ is an $\mathbb{R}$-linear function and $\varphi_{m}(m=1,2, \ldots, A)$ is a complex-valued function defined on

$$
S_{m}=\left\{\left(u_{m a}+i u_{m b}\right) \alpha_{m}+\sum_{\substack{j=1 \\ j \neq m}}^{A}\left(k_{j a}+i k_{j b}\right) \alpha_{j} \mid u_{m a}, u_{m b} \in \mathbb{N}_{0} ; k_{j a}, k_{j b} \in \mathbb{Z}\right\}\left(\supseteq S^{+}\right)
$$

satisfying

(1) $\varphi_{m}(0)=0$,

(2) $\varphi_{m}\left(x+\alpha_{j}\right)=\varphi_{m}\left(x+i \alpha_{j}\right)=\varphi_{m}(x)\left(j \neq m\right.$ and $\left.x \in S_{m}\right)$,

(3) $\varphi_{m}$ is uniformly continuous on $S_{m}$.

Proof. Let $f$ be a uniformly continuous solution of (2.2). There are three major steps in the proof. First, we show that the two limits $\lim _{N \rightarrow \infty} f\left(N \alpha_{m}\right) / N$ and $\lim _{N \rightarrow \infty} f\left(i N \alpha_{m}\right) / i N$ exist. Then we define an $\mathbb{R}$-linear function $\lambda: \mathbb{C} \rightarrow \mathbb{C}$ and show that $\lambda$ is uniformly continuous on $S^{+}$. Finally, the periodic functions $\varphi_{m}$ are constructed. 
Step 1. Let $\varepsilon>0$. By the uniform continuity of $f$, there exists $\delta>0$ such that

$$
\forall z, w \in S^{+}, \quad|z-w|<\delta \Longrightarrow|f(z)-f(w)|<\varepsilon .
$$

Since $S^{+}$is dense in $\mathbb{C}$, there are $x, y \in S^{+}$such that $|x-y|<\delta$. Write

$$
x=\sum_{m=1}^{A}\left(c_{m a}+i c_{m b}\right) \alpha_{m}, \quad y=\sum_{m=1}^{A}\left(d_{m a}+i d_{m b}\right) \alpha_{m},
$$

where all $c_{m a}, c_{m b}, d_{m a}, d_{m b} \in \mathbb{N}_{0}$. Set $q_{m \tau}=c_{m \tau}-d_{m \tau}(m=1,2, \ldots, A), \tau \in\{a, b\}$, and split $\{1,2, \ldots, A\}$ into any two disjoint nonempty sets $I$ and $J$ with $I \cup J=\{1,2, \ldots, A\}$.

We claim that for each $M \in \mathbb{N}$, we have

$$
\begin{aligned}
& \mid \sum_{t \in I}\left\{f\left(\left(c_{t a}+i c_{t b}\right) \alpha_{t}\right)-f\left(\left(d_{t a}+i d_{t b}\right) \alpha_{t}\right)\right\} \\
& \quad+\frac{1}{M} \sum_{j \in J} \eta_{j a}\left\{f\left(\left(w_{j a}+M\left|q_{j a}\right|\right) \alpha_{j}\right)-f\left(w_{j a} \alpha_{j}\right)\right\} \\
& \quad+\frac{1}{M} \sum_{j \in J} \eta_{j b}\left\{f\left(i\left(w_{j b}+M\left|q_{j b}\right|\right) \alpha_{j}\right)-f\left(i w_{j b} \alpha_{j}\right)\right\} \mid<\varepsilon,
\end{aligned}
$$

where, for $j \in J, w_{j a}, w_{j b}$ are any nonnegative integers, $\eta_{j \tau}=1$ if $q_{j \tau} \geq 0, \eta_{j \tau}=-1$ if $q_{j \tau}<0(\tau \in\{a, b\})$.

To prove this inequality, define $c_{j \tau}^{(k)}, d_{j \tau}^{(k)}$ for $\tau \in\{a, b\}, j \in J$, and $k \in \mathbb{N}$ as follows:

$$
\begin{gathered}
\text { if } q_{j \tau} \geq 0, \quad \text { set } c_{j \tau}^{(k)}=w_{j \tau}+k q_{j \tau}, \quad d_{j \tau}^{(k)}=w_{j \tau}+(k-1) q_{j \tau} ; \\
\text { if } q_{j \tau}<0, \quad \text { set } c_{j \tau}^{(k)}=w_{j \tau}+(k-1)\left|q_{j \tau}\right|, \quad d_{j \tau}^{(k)}=w_{j \tau}+k\left|q_{j \tau}\right| .
\end{gathered}
$$

We see that $c_{j \tau}^{(k)}, d_{j \tau}^{(k)} \in \mathbb{N}_{0}$ and $q_{j \tau}=c_{j \tau}^{(k)}-d_{j \tau}^{(k)}$. Now

$$
\begin{aligned}
& \left|\left\{\sum_{t \in I}\left(c_{t a}+i c_{t b}\right) \alpha_{t}+\sum_{j \in J}\left(c_{j a}^{(k)}+i c_{j b}^{(k)}\right) \alpha_{j}\right\}-\left\{\sum_{t \in I}\left(d_{t a}+i d_{t b}\right) \alpha_{t}+\sum_{j \in J}\left(d_{j a}^{(k)}+i d_{j b}^{(k)}\right) \alpha_{j}\right\}\right| \\
& \quad=\left|\sum_{m=1}^{A}\left(q_{m a}+i q_{m b}\right) \alpha_{m}\right|=\left|\sum_{m=1}^{A}\left(c_{m a}+i c_{m b}\right) \alpha_{m}-\sum_{m=1}^{A}\left(d_{m a}+i d_{m b}\right) \alpha_{m}\right|=|x-y|<\delta,
\end{aligned}
$$

which, by the uniform continuity condition (2.5) and the CFE (2.2), yields

$$
\begin{aligned}
& \mid \sum_{t \in I}\left\{f\left(\left(c_{t a}+i c_{t b}\right) \alpha_{t}\right)-f\left(\left(d_{t a}+i d_{t b}\right) \alpha_{t}\right)\right\} \\
& +\sum_{j \in J}\left\{f\left(c_{j a}^{(k)} \alpha_{j}\right)-f\left(d_{j a}^{(k)} \alpha_{j}\right)\right\}+\sum_{j \in J}\left\{f\left(i c_{j b}^{(k)} \alpha_{j}\right)-f\left(i d_{j b}^{(k)} \alpha_{j}\right)\right\} \mid<\varepsilon .
\end{aligned}
$$


In the last two sums, split $J$ into two pairs of disjoint subsets

$$
J=J_{a^{+}} \cup J_{a^{-}}, \quad J=J_{b^{+}} \cup J_{b^{-}},
$$

where $J_{a^{+}}=\left\{j \in J \mid q_{j a} \geq 0\right\}, J_{a^{-}}=\left\{j \in J \mid q_{j a}<0\right\}, J_{b^{+}}=\left\{j \in J \mid q_{j b} \geq 0\right\}$, and $J_{b^{-}}=$ $\left\{j \in J \mid q_{j b}<0\right\}$. Thus

$$
\begin{aligned}
\sum_{j \in J}\left\{f\left(c_{j a}^{(k)} \alpha_{j}\right)-f\left(d_{j a}^{(k)} \alpha_{j}\right)\right\}= & \sum_{j \in J_{a^{+}}}\left\{f\left(c_{j a}^{(k)} \alpha_{j}\right)-f\left(d_{j a}^{(k)} \alpha_{j}\right)\right\} \\
& +\sum_{j \in J_{a^{-}}}\left\{f\left(c_{j a}^{(k)} \alpha_{j}\right)-f\left(d_{j a}^{(k)} \alpha_{j}\right)\right\},
\end{aligned}
$$

and we also have a similar expression for $b$ in place of $a$ but with purely imginary arguments. Note that

$$
\begin{aligned}
& \sum_{k=1}^{M} \sum_{j \in J_{a^{+}}}\left\{f\left(c_{j a}^{(k)} \alpha_{j}\right)-f\left(d_{j a}^{(k)} \alpha_{j}\right)\right\} \\
& =\sum_{j \in J_{a^{+}}} \sum_{k=1}^{M}\left\{f\left(\left(w_{j a}+k q_{j a}\right) \alpha_{j}\right)-f\left(\left(w_{j a}+(k-1) q_{j a}\right) \alpha_{j}\right)\right\} \\
& =\sum_{j \in J_{a^{+}}}\left\{f\left(\left(w_{j a}+M q_{j a}\right) \alpha_{j}\right)-f\left(w_{j a} \alpha_{j}\right)\right\},
\end{aligned}
$$

and similarly,

$$
\begin{aligned}
& \sum_{k=1}^{M} \sum_{j \in J_{a^{-}}}\left\{f\left(c_{j a}^{(k)} \alpha_{j}\right)-f\left(d_{j a}^{(k)} \alpha_{j}\right)\right\}=\sum_{j \in J_{a^{-}}}\left\{f\left(w_{j a} \alpha_{j}\right)-f\left(\left(w_{j a}+M\left|q_{j a}\right|\right) \alpha_{j}\right)\right\}, \\
& \sum_{k=1}^{M} \sum_{j \in J_{b^{+}}}\left\{f\left(i c_{j b}^{(k)} \alpha_{j}\right)-f\left(i d_{j b}^{(k)} \alpha_{j}\right)\right\}=\sum_{j \in J_{b^{+}}}\left\{f\left(i\left(w_{j b}+M q_{j b}\right) \alpha_{j}\right)-f\left(i w_{j b} \alpha_{j}\right)\right\}, \\
& \sum_{k=1}^{M} \sum_{j \in J_{b^{-}}}\left\{f\left(i c_{j b}^{(k)} \alpha_{j}\right)-f\left(i d_{j b}^{(k)} \alpha_{j}\right)\right\}=\sum_{j \in J_{b^{-}}}\left\{f\left(i w_{j b} \alpha_{j}\right)-f\left(i\left(w_{j b}+M\left|q_{j b}\right|\right) \alpha_{j}\right)\right\} .
\end{aligned}
$$

Using these equations, summing over $k=1,2, \ldots, M$ in (2.10) and dividing by $M$, the claim (2.7) follows. For each $m$, by the denseness of $S^{+}$, there exist $q_{k a}, q_{k b} \in \mathbb{N}_{0}(k=$ $1,2, \ldots, A ; k \neq m)$ and $q_{m a}, q_{m b} \in \mathbb{N}$ such that $\left|\left(q_{1 a}+i q_{1 b}\right) \alpha_{1}+\cdots+\left(q_{A a}+i q_{A b}\right) \alpha_{A}\right|<\delta$, and so componentwise $\left|q_{1 a} \alpha_{1}+\cdots+q_{A a} \alpha_{A}\right|<\delta$ and $\left|i q_{1 b} \alpha_{1}+\cdots+i q_{A b} \alpha_{A}\right|<\delta$. From (2.2), (2.7) with $x=q_{1 a} \alpha_{1}+\cdots+q_{A a} \alpha_{A}, y=0, J=\{m\}$, and $I=\{1,2, \ldots, A\} \backslash\{m\}$, 
we deduce

$$
\left|\sum_{t \in I} f\left(q_{t a} \alpha_{t}\right)+\frac{1}{M}\left\{f\left(\left(w_{m a}+M q_{m a}\right) \alpha_{m}\right)-f\left(w_{m a} \alpha_{m}\right)\right\}\right|<\varepsilon,
$$

and similarly, with $x=i q_{1 b} \alpha_{1}+\cdots+i q_{A b} \alpha_{A}$, we deduce

$$
\left|\sum_{t \in I} f\left(i q_{t b} \alpha_{t}\right)+\frac{1}{M}\left\{f\left(i\left(w_{m b}+M q_{m b}\right) \alpha_{m}\right)-f\left(i w_{m b} \alpha_{m}\right)\right\}\right|<\varepsilon .
$$

For each $u, v \in \mathbb{N}$, by the division algorithm, we can write $u=w_{a}+M_{a} q_{m a}$ and $v=w_{b}+$ $M_{b} q_{m b}$, where $0 \leq w_{a}<q_{m a}$ and $0 \leq w_{b}<q_{m b}$. We see that if $u, v \rightarrow \infty$, then $M_{a}$ and $M_{b} \rightarrow$ $\infty$. This allows us to consider in (2.15) and (2.16) the values of $w_{m a}$ and $w_{m b}$ in the same ranges as $w_{a}$ and $w_{b}$, respectively. Letting $M \rightarrow \infty$ in (2.15), we have

$$
\left|\sum_{t \in I} f\left(q_{t a} \alpha_{t}\right)+q_{m a} L\right| \leq \varepsilon
$$

where $L$ is any one of the possible limits of the sequence $\left\{f\left(u \alpha_{m}\right) / u\right\}$.

We now show that $L$ is unique. Suppose that $L_{1}, L_{2}$ are any two limits of the sequence. From (2.17), we get $\left|q_{m a}\right|\left|L_{1}-L_{2}\right| \leq 2 \varepsilon$, and so $L_{1}=L_{2}$. This implies that $\lim _{N \rightarrow \infty} f\left(N \alpha_{m}\right) /$ $N=\lambda_{m R}$ exists. The existence of $\lim _{N \rightarrow \infty} f\left(i N \alpha_{m}\right) / i N=\lambda_{m I}$ is similarly derived from (2.16).

Step 2. For each $x \in \mathbb{C}$, if $x=\sum_{m=1}^{A}\left(x_{m a}+i x_{m b}\right) \alpha_{m}\left(x_{m a}, x_{m b} \in \mathbb{R}\right)$, define $\lambda(x)=$ $\sum_{m=1}^{A}\left(x_{m a} \lambda_{m R}+i x_{m b} \lambda_{m I}\right)$. The representation of any $x \in \mathbb{C}$ with respect to $\alpha_{1}, \alpha_{2}, \ldots, \alpha_{A}$ above is clearly always possible but certainly not unique. We first verify that $\lambda$ is indeed well defined. To do so, it suffices to show that if $0=\sum_{m=1}^{A}\left(x_{m a}+i x_{m b}\right) \alpha_{m}$, then $\sum_{m=1}^{A}\left(x_{m a} \lambda_{m R}+i x_{m b} \lambda_{m I}\right)=0$. There are two possible cases.

Case 1. $x_{m \tau}=0$ for all $m \in\{1,2, \ldots, A\}$ and all $\tau \in\{a, b\}$. This case is trivial.

Case 2. There exists $x_{m^{\prime} \tau^{\prime}} \neq 0$ for some $m^{\prime} \in\{1,2, \ldots, A\}$ and $\tau^{\prime} \in\{a, b\}$.

From Dirichlet's diophantine approximation theorem (see [5, Chapter I]), for each $\nu \in \mathbb{N}$, there are $t^{(v)}, k_{m \tau}^{(\nu)} \in \mathbb{Z}$ with $t^{(v)}>0$ such that

$$
\left|t^{(\nu)} x_{m \tau}-k_{m \tau}^{(v)}\right|<\frac{1}{v} \quad(m=1,2, \ldots, A ; \tau=a, b),
$$

with $k_{m \tau}^{(\nu)}:=0$ if $x_{m \tau}=0$. Note that we may choose $t^{(\nu)} \rightarrow \infty$ as $\nu \rightarrow \infty$, so that for $x_{m \tau} \neq 0$, we must have $\left|k_{m \tau}^{(\nu)}\right| \rightarrow \infty$ as $\nu \rightarrow \infty$. From the representation of 0 , we get

$$
\begin{aligned}
\left|\sum_{m=1}^{A}\left(k_{m a}^{(v)}+i k_{m b}^{(v)}\right) \alpha_{m}\right| & =\left|\sum_{m=1}^{A}\left(k_{m a}^{(v)}+i k_{m b}^{(v)}\right) \alpha_{m}-t^{(\nu)} \sum_{m=1}^{A}\left(x_{m a}+i x_{m b}\right) \alpha_{m}\right| \\
& =\left|\sum_{m=1}^{A}\left\{\left(k_{m a}^{(v)}-t^{(\nu)} x_{m a}\right)+i\left(k_{m b}^{(v)}-t^{(v)} x_{m b}\right)\right\} \alpha_{m}\right| \leq \frac{2}{v} \sum_{m=1}^{A}\left|\alpha_{m}\right| .
\end{aligned}
$$


Thus

$$
\lim _{\nu \rightarrow \infty}\left|\sum_{m=1}^{A}\left(k_{m a}^{(\nu)}+i k_{m b}^{(v)}\right) \alpha_{m}\right|=0 .
$$

From (2.18), using the fact that $x_{m^{\prime} \tau^{\prime}} \neq 0$, we arrive at

$$
\lim _{\nu \rightarrow \infty} \frac{k_{m \tau}^{(\nu)}}{k_{m^{\prime} \tau^{\prime}}^{(v)}}=\frac{x_{m \tau}}{x_{m^{\prime} \tau^{\prime}}}
$$

Clearly, for each $m=1,2, \ldots, A$, we have $\operatorname{sgn} k_{m \tau}^{(v)}=\operatorname{sgn} x_{m \tau}$ when $v$ is sufficiently large. Let $U_{a}^{+}=\left\{m \mid x_{m a}>0\right\}, U_{a}^{-}=\left\{m \mid x_{m a}<0\right\}, U_{b}^{+}=\left\{m \mid x_{m b}>0\right\}$, and $U_{b}^{-}=\left\{m \mid x_{m b}<0\right\}$. Rewriting (2.20) as

$$
\lim _{\nu \rightarrow \infty}\left|\left(\sum_{t \in U_{a}^{+}} k_{t a}^{(\nu)} \alpha_{t}+\sum_{t \in U_{b}^{+}} i k_{t b}^{(\nu)} \alpha_{t}\right)-\left(\sum_{s \in U_{a}^{-}}\left|k_{s a}^{(v)}\right| \alpha_{s}+\sum_{s \in U_{b}^{-}} i\left|k_{s b}^{(\nu)}\right| \alpha_{s}\right)\right|=0,
$$

using uniform continuity, (2.2), and $f(0)=0$, we get as $\nu \rightarrow \infty$,

$$
\left|\left\{\sum_{t \in U_{a}^{+}} f\left(k_{t a}^{(v)} \alpha_{t}\right)+\sum_{t \in U_{b}^{+}} f\left(i k_{t b}^{(v)} \alpha_{t}\right)\right\}-\left\{\sum_{s \in U_{a}^{-}} f\left(\left|k_{s a}^{(v)}\right| \alpha_{s}\right)+\sum_{s \in U_{b}^{-}} f\left(i\left|k_{s b}^{(v)}\right| \alpha_{s}\right)\right\}\right| \longrightarrow 0,
$$

and so

$$
\begin{aligned}
& \mid \sum_{t \in U_{a}^{+}} \frac{f\left(k_{t a}^{(v)} \alpha_{t}\right)}{k_{m^{\prime} \tau^{\prime}}^{(\nu)}} \cdot \frac{k_{t a}^{(v)}}{k_{t a}^{(v)}}+\sum_{t \in U_{b}^{+}} \frac{f\left(i k_{t b}^{(\nu)} \alpha_{t}\right)}{k_{m^{\prime} \tau^{\prime}}^{(\nu)}} \cdot \frac{i k_{t b}^{(v)}}{i k_{t b}^{(v)}} \\
& \quad-\sum_{s \in U_{a}^{-}} \frac{f\left(\left|k_{s a}^{(\nu)}\right| \alpha_{s}\right)}{k_{m^{\prime} \tau^{\prime}}^{(\nu)}} \cdot \frac{\left|k_{s a}^{(\nu)}\right|}{\left|k_{s a}^{(\nu)}\right|}-\sum_{s \in U_{b}^{-}} \frac{f\left(i\left|k_{s b}^{(\nu)}\right| \alpha_{s}\right)}{k_{m^{\prime} \tau^{\prime}}^{(v)}} \cdot \frac{i\left|k_{s b}^{(\nu)}\right|}{i\left|k_{s b}^{(\nu)}\right|} \mid \longrightarrow 0,
\end{aligned}
$$

which, by (2.21) and Step 1, yields

$$
\left|\sum_{t \in U_{a}^{+}} \lambda_{t R} \frac{x_{t a}}{x_{m^{\prime} \tau^{\prime}}}+\sum_{t \in U_{b}^{+}} \lambda_{t I} \frac{i x_{t b}}{x_{m^{\prime} \tau^{\prime}}}-\sum_{s \in U_{a}^{-}} \lambda_{s R} \frac{\left|x_{s a}\right|}{x_{m^{\prime} \tau^{\prime}}}-\sum_{s \in U_{b}^{-}} \lambda_{s I} \frac{i\left|x_{s b}\right|}{x_{m^{\prime} \tau^{\prime}}}\right|=0 .
$$

Thus $\sum_{m=1}^{A}\left(x_{m a} \lambda_{m R}+i x_{m b} \lambda_{m I}\right)=0$, that is, $\lambda(0)=0$, which shows that $\lambda$ is a function. That $\lambda$ is linear over $\mathbb{R}$, and so uniformly continuous on $S^{+}$, is easily checked.

Step 3. Define the function $\omega: S^{+} \rightarrow \mathbb{C}$ by $\omega(x)=f(x)-\lambda(x)$.

Then $\omega$ is uniformly continuous and satisfies (2.2) on $S^{+}$as $f$ and $\lambda$ are. Moreover, by Step 1 and the linearity of $\lambda$, we have

$$
\lim _{N \rightarrow \infty} \frac{\omega\left(N \alpha_{m}\right)}{N}=0=\lim _{N \rightarrow \infty} \frac{\omega\left(i N \alpha_{m}\right)}{i N} .
$$


For each $m=1,2, \ldots, A$, define $\varphi_{m}: S_{m}\left(\supseteq S^{+}\right) \rightarrow \mathbb{C}$ by

(1) $\varphi_{m}(0)=0$,

(2) $\varphi_{m}\left(x+\alpha_{j}\right)=\varphi_{m}\left(x+i \alpha_{j}\right)=\varphi_{m}(x)$ for all $j \neq m, x \in S_{m}$,

(3) $\varphi_{m}\left(\left(u_{m a}+i u_{m b}\right) \alpha_{m}\right)=\omega\left(\left(u_{m a}+i u_{m b}\right) \alpha_{m}\right)$.

To confirm the shape of solution, note that for $x=\sum_{m=1}^{A}\left(x_{m a}+i x_{m b}\right) \alpha_{m} \in S^{+}$, we have

$$
\begin{aligned}
f(x) & =\lambda(x)+\omega(x)=\lambda(x)+\sum_{m=1}^{A} \omega\left(\left(x_{m a}+i x_{m b}\right) \alpha_{m}\right) \\
& =\lambda(x)+\sum_{m=1}^{A} \varphi_{m}\left(\left(x_{m a}+i x_{m b}\right) \alpha_{m}\right)=\lambda(x)+\sum_{m=1}^{A} \varphi_{m}(x) .
\end{aligned}
$$

Finally, to complete the proof, we are left only to show that $\varphi_{m}$ is uniformly continuous on $S_{m}$. Fix $m$ and let $\varepsilon>0$. Since $\omega$ is uniformly continuous on $S^{+}$, there exists $\delta^{*}>0$ such that for all $x, y \in S^{+},|x-y|<\delta^{*} \Rightarrow|\omega(x)-\omega(y)|<\varepsilon$. Let

$$
\zeta=\left(u_{m a}+i u_{m b}\right) \alpha_{m}+\sum_{\substack{j=1 \\ j \neq m}}^{A}\left(k_{j a}+i k_{j b}\right) \alpha_{j}, \quad \eta=\left(v_{m a}+i v_{m b}\right) \alpha_{m}+\sum_{\substack{j=1 \\ j \neq m}}^{A}\left(l_{j a}+i l_{j b}\right) \alpha_{j}
$$

be elements of $S_{m}$ with $|\zeta-\eta|<\delta^{*}$. For each $j \neq m$, rewrite $q_{j a}+i q_{j b}=\left(k_{j a}+i k_{j b}\right)-$ $\left(l_{j a}+i l_{j b}\right)$. Choose $u_{j a}, u_{j b}, v_{j a}, v_{j b} \in \mathbb{N}_{0}$ so that $q_{j a}+i q_{j b}=\left(u_{j a}+i u_{j b}\right)-\left(v_{j a}+i v_{j b}\right)$. Let $x=\left(u_{m a}+i u_{m b}\right) \alpha_{m}+\sum_{j=1, j \neq m}^{A}\left(u_{j a}+i u_{j b}\right) \alpha_{j}$, and $y=\left(v_{m a}+i v_{m b}\right) \alpha_{m}+\sum_{j=1, j \neq m}^{A}\left(v_{j a}+\right.$ $\left.i v_{j b}\right) \alpha_{j}$. Then

$$
\begin{aligned}
|x-y| & =\left|\left\{\left(u_{m a}+i u_{m b}\right) \alpha_{m}-\left(v_{m a}+i v_{m b}\right) \alpha_{m}\right\}+\sum_{\substack{j=1 \\
j \neq m}}^{A}\left(q_{j a}+i q_{j b}\right) \alpha_{j}\right| \\
& =\left|\left\{\left(u_{m a}+i u_{m b}\right) \alpha_{m}-\left(v_{m a}+i v_{m b}\right) \alpha_{m}\right\}+\sum_{\substack{j=1 \\
j \neq m}}^{A}\left(\left(k_{j a}+i k_{j b}\right)-\left(l_{j a}+i l_{j b}\right)\right) \alpha_{j}\right| \\
& =|\zeta-\eta|<\delta^{*} .
\end{aligned}
$$

Applying (2.7) with $f=\omega, I=\{m\}, J=\{1,2, \ldots, A\} \backslash\{m\}, w_{j a}=w_{j b}=0$, and $q_{j \tau}=u_{j \tau}-$ $v_{j \tau}(\tau=a, b)$, we get

$$
\begin{aligned}
& \mid \omega\left(\left(u_{m a}+i u_{m b}\right) \alpha_{m}\right)-\omega\left(\left(v_{m a}+i v_{m b}\right) \alpha_{m}\right) \\
& +\frac{1}{M} \sum_{j \in J} \eta_{j a}\left\{\omega\left(M\left|q_{j a}\right| \alpha_{j}\right)-0\right\}+\frac{1}{M} \sum_{j \in J} \eta_{j b}\left\{\omega\left(i M\left|q_{j b}\right| \alpha_{j}\right)-0\right\} \mid<\varepsilon .
\end{aligned}
$$


Since the sequences $\omega\left(M\left|q_{j a}\right| \alpha_{j}\right) / M$ and $\omega\left(i M\left|q_{j b}\right| \alpha_{j}\right) / M$ are subsequences of $\omega\left(N \alpha_{j}\right) / N$ and $\omega\left(i N \alpha_{j}\right) / N$, respectively, by (2.26), we have

$$
\lim _{M \rightarrow \infty} \frac{\omega\left(M\left|q_{j a}\right| \alpha_{j}\right)}{M}=0=\lim _{M \rightarrow \infty} \frac{\omega\left(i M\left|q_{j b}\right| \alpha_{j}\right)}{M} \quad(j \in J)
$$

Letting $M \rightarrow \infty$ in (2.30) and using (2.31), we get

$$
\left|\omega\left(\left(u_{m a}+i u_{m b}\right) \alpha_{m}\right)-\omega\left(\left(v_{m a}+i v_{m b}\right) \alpha_{m}\right)\right| \leq \varepsilon .
$$

As $\varphi_{m}(\zeta)=\varphi_{m}\left(\left(u_{m a}+i u_{m b}\right) \alpha_{m}\right)=\omega\left(\left(u_{m a}+i u_{m b}\right) \alpha_{m}\right)$ and similarly $\varphi_{m}(\eta)=\omega\left(\left(v_{m a}+\right.\right.$ $\left.\left.i v_{m b}\right) \alpha_{m}\right)$, we deduce that $\left|\varphi_{m}(\zeta)-\varphi_{m}(\eta)\right| \leq \varepsilon$, that is, $\varphi_{m}$ is uniformly continuous on $S_{m}$, which completes the proof.

Corollary 2.2. Let $A \in \mathbb{N}, A \geq 2$, and $\alpha_{1}, \alpha_{2}, \ldots, \alpha_{A} \in \mathbb{R}$ be such that

(1) $S^{+}=\left\{\sum_{m=1}^{A}\left(u_{m}+i v_{m}\right) \alpha_{m} \mid u_{m}, v_{m} \in \mathbb{N}_{0}\right\}$ is dense in $\mathbb{C}$,

(2) $T_{m}=\left\{\sum_{j=1, j \neq m}^{A}\left(u_{j}+i v_{j}\right) \alpha_{j} \mid u_{j}, v_{j} \in \mathbb{Z}\right\}(m=1, \ldots, A)$ is dense in $S_{m}$.

If $f: S^{+} \rightarrow \mathbb{C}$ is a uniformly continuous solution of the CFE (2.2), then $f$ is an $\mathbb{R}$-linear function; in particular $f(z)=a z+b \bar{z}$, where $a, b$ are arbitrary complex constants and $\bar{z}$ denotes the complex conjugate of $z$.

Proof. The first part will follow from Theorem 2.1 if we show that each $\varphi_{m}$ vanishes identically. This is immediate from the facts that elements of $S_{m}$ can be approximated arbitrarily closely by elements of $T_{m}$, while $\varphi_{m}$ is continuous on $S_{m}$ and vanishes identically on $T_{m}$. The second part follows from the linearity of $\lambda$, viz, if $z=x+i y \in \mathbb{C}$, then $f(z)=\lambda(z)=x \lambda(1)+y \lambda(i)$.

Corollary 2.2 may be regarded as an extension of a special case of the classical complex CFE: $f\left(z_{1}+z_{2}\right)=f\left(z_{1}\right)+f\left(z_{2}\right)$, whose general solution is of the form $f(z)=a z+b \bar{z}$; see [1, Proposition 2 in Chapter 5].

The next two propositions provide examples for Theorem 2.1 and Corollary 2.2. For convenience, we make use of the following notation: $\mathbb{Z}[i]$ denotes the ring of Gaussian integers, while $\mathbb{N}_{0}[i]$ denotes the subset of Gaussian integers both of whose real and imaginary parts are nonnegative.

Proposition 2.3. Let $A \in \mathbb{N}, A \geq 2$, and $\alpha_{1}, \alpha_{2}, \ldots, \alpha_{A} \in \mathbb{R}$. If there are two rationally independent $\alpha_{j}^{\prime}$ s whose ratio is a negative real number, then $S^{+}:=\alpha_{1} \mathbb{N}_{0}[i]+\alpha_{2} \mathbb{N}_{0}[i]+\cdots+$ $\alpha_{A} \mathbb{N}_{0}[i]$ is dense in $\mathbb{C}$.

Proof. Suppose that $\alpha_{m}$ and $\alpha_{n}$ are rationally independent and $\alpha_{m} / \alpha_{n}<0$. Then both are nonzero and at least one of them is irrational. Consider here the case where $\alpha_{m}$ is irrational $>0$ and $\alpha_{n}<0$; other cases can be similarly handled. Let $r_{1}+i r_{2} \in \mathbb{C}$ and $\epsilon>0$. By Kronecker's theorem, see [5, Chapter II], there are infinitely many natural numbers $u_{1}, v_{1}$ and integers $u_{2}, v_{2}$ such that $\left|u_{1}\left(\alpha_{m} /\left|\alpha_{n}\right|\right)-u_{2}-r_{1} /\right| \alpha_{n}||<\varepsilon / 2\left|\alpha_{n}\right|, \mid v_{1}\left(\alpha_{m} /\left|\alpha_{n}\right|\right)-v_{2}-$ $r_{2} /\left|\alpha_{n}\right||<\varepsilon / 2| \alpha_{n} \mid$. We can choose these integers so that $u_{1} \alpha_{m}-r_{1}>0, v_{1} \alpha_{m}-r_{2}>0$, and so $u_{2}, v_{2}$ are also positive. Thus $\left|\left(u_{1}+i v_{1}\right) \alpha_{m}+\left(u_{2}+i v_{2}\right) \alpha_{n}-\left(r_{1}+i r_{2}\right)\right| \leq \mid u_{1} \alpha_{m}+u_{2} \alpha_{n}-$ $r_{1}|+| v_{1} \alpha_{m}+v_{2} \alpha_{n}-r_{2} \mid<\varepsilon$, yielding the denseness of $S^{+}$in $\mathbb{C}$. 
Let us note in passing that in Proposition 2.3, denseness only requires rational independence of two base elements, which to a certain extent indicates that the two concepts, denseness and rational independence, are not totally equivalent.

Proposition 2.4. Let $A \in \mathbb{N}, A \geq 3$, and $\alpha_{1}, \alpha_{2}, \ldots, \alpha_{A} \in \mathbb{R}$. If the $\alpha_{j}^{\prime} s$ are pairwise rationally independent, then each $T_{m}=\sum_{j=1, j \neq m}^{n} \alpha_{j} \mathbb{Z}[i](m=1, \ldots, A)$ is dense in $\mathbb{C}$.

Proof. It suffices to show that $\alpha_{k} \mathbb{Z}[i]+\alpha_{l} \mathbb{Z}[i](l, k \neq m)$ is dense in $\mathbb{C}$. Let $r_{1}+i r_{2} \in \mathbb{C}$ and $\epsilon>0$. Again by Kronecker's theorem, there are infinitely many $u_{1}, v_{1} \in \mathbb{N}$ and $u_{2}, v_{2} \in \mathbb{Z}$ such that $\left|u_{1}\left(\alpha_{k} / \alpha_{l}\right)-u_{2}-r_{1} / \alpha_{l}\right|<\varepsilon / 2\left|\alpha_{l}\right|$ and $\left|v_{1}\left(\alpha_{k} / \alpha_{l}\right)-v_{2}-r_{2} / \alpha_{l}\right|<\varepsilon / 2\left|\alpha_{l}\right|$. Thus $\left|\left(u_{1}+i v_{1}\right) \alpha_{k}+\left(u_{2}+i v_{2}\right) \alpha_{l}-\left(r_{1}+i r_{2}\right)\right|<\varepsilon$.

The combined result of Corollary 2.2 and Proposition 2.4 can be regarded as a complex counterpart to [8, Theorem 2.1], which states roughly that for $A=3, n=1$, if $\alpha_{1}$, $\alpha_{2}, \alpha_{3}$ are positive and all the possible ratios $\alpha_{k} / \alpha_{j}(k \neq j)$ are irrational, then increasing functions which are solutions of the CFE (1.1) are first degree monomials.

Comparing the condition of " $\mathbb{Q}$-linearly independence" in Pisot and Schoenberg (see [9]) with our condition of being "dense in $\mathbb{C}$," a natural question is whether they are related. The following examples show otherwise.

Example 2.5. (1) Let $\alpha_{1}=1, \alpha_{2}=\sqrt{2}$. Clearly, both are $\mathbb{Q}$-linearly independent. Each element of $S^{+}$, as defined in Theorem 2.1, is of the form $\left(u_{1}+u_{2} \sqrt{2}\right)+i\left(v_{1}+v_{2} \sqrt{2}\right)$, whose real and imaginary parts are nonnegative real numbers, which shows that $S^{+}$cannot be dense in $\mathbb{C}$.

(2) Let $\alpha_{1}=1, \alpha_{2}=\sqrt{2}$ and $\alpha_{3}=-\sqrt{2}$. Then $\alpha_{1}, \alpha_{2}, \alpha_{3}$ are $\mathbb{Q}$-linearly dependent, and by Proposition 2.3, the corresponding set $S^{+}$is dense in $\mathbb{C}$.

(3) Let $\alpha_{1}=1, \alpha_{2}=-\sqrt{2}$. Then $\alpha_{1}, \alpha_{2}$ are $\mathbb{Q}$-linearly independent, and the corresponding $S^{+}$is dense in $\mathbb{C}$ by Proposition 2.3.

(4) Let $\alpha_{1}=\sqrt{2}, \alpha_{2}=-\sqrt{2}$. Then $\alpha_{1}, \alpha_{2}$ are $\mathbb{Q}$-linearly dependent and the corresponding $S^{+}$is not dense in $\mathbb{C}$.

\section{Case of complex base elements}

Using the same proofs as in Theorem 2.1 and Corollary 2.2, solutions of similar CFE with complex base elements $\alpha_{j}$ and rational integer coefficients can accordingly be derived. We merely state the results omitting the proofs.

Theorem 3.1. Let $A \in \mathbb{N}, A \geq 3$, and $\alpha_{1}, \alpha_{2}, \ldots, \alpha_{A} \in \mathbb{C}$ be such that the set $R^{+}:=$ $\left\{\sum_{m=1}^{A} u_{m} \alpha_{m} \mid u_{m} \in \mathbb{N}_{0}\right\}$ is dense in $\mathbb{C}$. If $f: R^{+} \rightarrow \mathbb{C}$ is a uniformly continuous function satisfying

$$
f\left(\sum_{m=1}^{A} u_{m} \alpha_{m}\right)=\sum_{m=1}^{A} f\left(u_{m} \alpha_{m}\right),
$$

then $f$ can be uniquely written as $f(x)=\lambda(x)+\sum_{m=1}^{A} \varphi_{m}(x)$, where $\lambda: \mathbb{C} \rightarrow \mathbb{C}$ is an $\mathbb{R}$ linear function and each $\varphi_{m}(m=1,2, \ldots, A)$ is a complex-valued function defined on $R_{m}:=$ 
$\left\{u_{m} \alpha_{m}+\sum_{j=1, j \neq m}^{A} k_{j} \alpha_{j} \mid u_{m} \in \mathbb{N}_{0} ; k_{j} \in \mathbb{Z}\right\}\left(\supseteq R^{+}\right)$satisfying

(1) $\varphi_{m}(0)=0$,

(2) $\varphi_{m}\left(x+\alpha_{j}\right)=\varphi_{m}(x)\left(j \neq m\right.$ and $\left.x \in R_{m}\right)$,

(3) $\varphi_{m}$ is uniformly continuous on $R_{m}$.

Corollary 3.2. Let the hypotheses be as in Theorem 3.1 but assume further that $A \geq 3$ and $R_{m}^{\prime}:=\left\{\sum_{j=1, j \neq m}^{A} u_{j} \alpha_{j} \mid u_{j} \in \mathbb{Z}\right\}(m=1, \ldots, A)$ is dense in $R_{m}$. If $f: R^{+} \rightarrow \mathbb{C}$ is a uniformly continuous solution of (3.1), then $f$ is an $\mathbb{R}$-linear function; in particular, $f(z)=a z+b \bar{z}$, where $a, b$ are arbitrary complex constants and $\bar{z}$ denotes the complex conjugate of $z$.

The next proposition gives us classes of examples for Theorem 3.1 and Corollary 3.2.

Proposition 3.3. Let $a, b, q_{1}, q_{2}, q_{3}$, and $q_{4}$ be real numbers

(1) If $1, q_{1} / a, q_{2} / b$ are rationally independent, then $a \mathbb{Z}+i b \mathbb{Z}+\left(q_{1}+i q_{2}\right) \mathbb{Z}$ is dense in $\mathbb{C}$.

(2) If $1, q_{1} / a, q_{2} / b$ are rationally independent and $q_{1} / a, q_{2} / b,-a b$ are all negative, then $a \mathbb{N}_{0}+i b \mathbb{N}_{0}+\left(q_{1}+i q_{2}\right) \mathbb{N}_{0}$ is dense in $\mathbb{C}$.

(3) If $q_{3} / a, q_{4} / b \in \mathbb{R} \backslash \mathbb{Q}$, then $a \mathbb{Z}+i b \mathbb{Z}+q_{3} \mathbb{Z}+i q_{4} \mathbb{Z}$ is dense in $\mathbb{C}$.

(4) If $q_{3} / a, q_{4} / b \in \mathbb{R}^{-} \backslash \mathbb{Q}$ and $a b>0$, then $a \mathbb{N}_{0}+i b \mathbb{N}_{0}+q_{3} \mathbb{N}_{0}+i q_{4} \mathbb{N}_{0}$ is dense in $\mathbb{C}$.

(5) If (i) $1, q_{1} / a, q_{2} / b$ are rationally independent,

(ii) $1, q_{1} / q_{3}, q_{2} / q_{4}$ are rationally independent,

(iii) $q_{3} / a, q_{4} / b \in \mathbb{R} \backslash \mathbb{Q}$,

(iv) either $q_{1} / a, q_{2} / b,-a b$ or $q_{3} / a, q_{4} / b,-a b$ are all negative,

then $a \mathbb{N}_{0}+i b \mathbb{N}_{0}+\left(q_{1}+i q_{2}\right) \mathbb{N}_{0}+q_{3} \mathbb{N}_{0}+i q_{4} \mathbb{N}_{0}$ is dense in $\mathbb{C}$.

Furthermore, $\alpha_{1}=a, \alpha_{2}=i b, \alpha_{3}=q_{1}+i q_{2}, \alpha_{4}=q_{3}, \alpha_{5}=i q_{4}$ satisfy the conditions set out in Corollary 3.2.

Proof. Let $r+i s \in \mathbb{C}$ and $\epsilon>0$.

(1) By the multidimentional Kronecker theorem, see [5, Chapter 2], there are infinitely many $n \in \mathbb{N}$ and $p_{1}, p_{2} \in \mathbb{Z}$ such that $\left|n\left(q_{1} / a\right)-p_{1}-r / a\right|<\epsilon /|a|$ and $\left|n\left(q_{2} / b\right)-p_{2}-s / b\right|<\epsilon /|b|$. Hence $\left|-p_{1}(a)-p_{2}(i b)+n\left(q_{1}+i q_{2}\right)-(r+i s)\right|<2 \epsilon$.

(2) By Kronecker's theorem, there are infinitely many integers $n \in \mathbb{N}, p_{1}, p_{2} \in \mathbb{Z}$ such that $\left|n\left(q_{1} /|a|\right)-p_{1}-r /\right| a||<\epsilon /|a|$ and $\left|n\left(q_{2} /|b|\right)-p_{2}-s /\right| b||<\epsilon /|b|$. Clearly, we can choose them in such a way that both $n\left(q_{1} /|a|\right)-r /|a|$ and $n\left(q_{2} /|b|\right)-$ $s /|b|>0$, which makes $p_{1}, p_{2}$ positive.

The proofs of (3) and (4) are analogous to those of (1) and (2), respectively.

(5) The former part follows from (2) while the latter follows readily from (1) and (3).

The condition of $R^{+}$being dense in $\mathbb{C}$ implies that the base elements $\alpha_{j}^{\prime} s$ neccessarily span the $\mathbb{R}$-vector space $\mathbb{C}$ as followed from the next proposition.

Proposition 3.4. Let $\alpha_{1}, \alpha_{2}, \ldots, \alpha_{A} \in \mathbb{C}$. If $R^{+}:=\left\{\sum_{m=1}^{A} u_{m} \alpha_{m} \mid u_{m} \in \mathbb{N}_{0}\right\}$ is dense in $\mathbb{C}$, then there exist $k \neq l$ such that $\alpha_{k}, \alpha_{l}$ are $\mathbb{R}$-linear independent.

Proof. Assume, without loss of generality, that each $\alpha_{m} \neq 0$. Suppose that each pair of $\alpha_{j}^{\prime} s$ is $\mathbb{R}$-linearly dependent. Then there are $r_{k} \in \mathbb{R}-\{0\}$ such that $\alpha_{k}=r_{k} \alpha_{1}(k=2, \ldots, A)$. Hence $\mathbb{R}^{+}=\alpha_{1}\left[\mathbb{N}_{0}+r_{2} \mathbb{N}_{0}+\cdots+r_{A} \mathbb{N}_{0}\right]$ which cannot be dense in $\mathbb{C}$. 
As in the case of real base elements, the notions of " $\mathbb{Q}$-linear independence" and "denseness" are not equivalent in the complex base elements case either, as seen in the following examples.

Example 3.5. (1) Let $\alpha_{1}=1+i \sqrt{2}, \alpha_{2}=1+i \sqrt{3}$. Each element of $R^{+}$, as defined in Theorem 3.1 , is of the form $(u+v)+i(u \sqrt{+} v \sqrt{3})$, whose real part is an integer which makes $R^{+}$not dense in $\mathbb{C}$, while $\alpha_{1}$ and $\alpha_{2}$ are clearly $\mathbb{Q}$-linearly independent.

(2) Let $\alpha_{1}=-1, \alpha_{2}=-i, \alpha_{3}=\sqrt{2}+i \sqrt{3}$, and $\alpha_{4}=1+i$. Clearly, $\alpha_{1}, \alpha_{2}, \alpha_{3}$ are $\mathbb{Q}$ linearly dependent. By Proposition 3.3(2), $R^{+}$is dense in $\mathbb{C}$.

(3) Let $\alpha_{1}=-1, \alpha_{2}=-i$, and $\alpha_{3}=\sqrt{2}+i \sqrt{3}$. By Proposition 3.3(2), $R^{+}$is dense in $\mathbb{C}$, and $\alpha_{1}, \alpha_{2}, \alpha_{3}$ are $\mathbb{Q}$-linearly independent.

(4) Let $\alpha_{1}=-1, \alpha_{2}=-i$, and $\alpha_{3}=1+i$. Here $\alpha_{1}, \alpha_{2}, \alpha_{3}$ are $\mathbb{Q}$-linearly dependent and $R^{+}$is not dense in $\mathbb{C}$.

We end this paper with two final remarks.

(i) In Theorem 3.1, the number of base elements $\alpha^{\prime} s$ which makes $R^{+}$dense in $\mathbb{C}$ cannot be fewer than three. This is seen by noting that any set $R^{+}$generated by two $\mathbb{R}$-linearly independent base elements is made up only of lattice points spanned by the two $\alpha^{\prime} s$, and is clearly not dense in $\mathbb{C}$. This fact was already mentioned in [9, page 130].

(ii) At the beginning of [8], a question similar to an old theorem of Erdös [4] (see also $[2,3,6,7,10,11])$ is posed whether the functional equation $F\left(p_{1}^{u_{1}} \cdots p_{k}^{u_{k}}\right)=F\left(p_{1}^{u_{1}}\right)+$ $\cdots+F\left(p_{k}^{u_{k}}\right)$, where $p_{1}, \ldots, p_{k}$ are distinct primes and $u_{1}, \ldots, u_{k}$ are natural numbers, under some natural conditions such as monotonicity or continuity, has only solutions of the form $F(n)=C \log n$. Using the transformation $F\left(e^{z}\right)=f(z)$, Theorem 3.1 and Corollary 3.2 provide some answers to their complex counterparts.

\section{References}

[1] J. Aczél and J. Dhombres, Functional Equations in Several Variables, Encyclopedia of Mathematics and Its Applications, vol. 31, Cambridge University Press, Cambridge, 1989.

[2] A. S. Besicovitch, On additive functions of a positive integer, Studies in Mathematical Analysis and Related Topics, Stanford University Press, California, 1962, pp. 38-41, Essays in honour of G. Pólya.

[3] J. M. Cohen, Monotone multiplicative functions, The American Mathematical Monthly 96 (1989), no. 6, 512-513.

[4] P. Erdös, On the distribution function of additive functions, Annals of Mathematics 47 (1946), $1-20$.

[5] E. Hlawka, J. Schoissengeier, and R. Taschner, Geometric and Analytic Number Theory, Universitext, Springer, Berlin, 1991.

[6] K. Kovács, On the characterization of additive functions with monotonic norm, Journal of Number Theory 24 (1986), no. 3, 298-304.

[7] L. Moser and J. Lambek, On monotone multiplicative functions, Proceedings of the American Mathematical Society 4 (1953), 544-545.

[8] Ch. Pisot and I. J. Schoenberg, Arithmetic problems concerning Cauchy's functional equation, Illinois Journal of Mathematics 8 (1964), 40-56.

[9] - Arithmetic problems concerning Cauchy's functional equation. II, Illinois Journal of Mathematics 9 (1965), 129-136. 


\section{Cauchy's functional equation}

[10] E. Wirsing, Characterisation of the logarithm as an additive function, Number Theory Institute (Proceedings of Symposia in Pure Mathe., Vol. XX, State University of New York, Stony Brook, NY, 1969), American Mathematical Society, Rhode Island, 1971, pp. 375-381.

[11] E. Wirsing and D. Zagier, Multiplicative functions with difference tending to zero, Acta Arithmetica 100 (2001), no. 1, 75-78.

Watcharapon Pimsert: Department of Mathematics, Chulalongkorn University, Bangkok 10330,

Thailand

E-mail address: watcharapon.pi@student.chula.ac.th

Vichian Laohakosol: Department of Mathematics, Kasetsart University, Bangkok 10900, Thailand E-mail address: fscivil@ku.ac.th

Sajee Pianskool: Department of Mathematics, Chulalongkorn University, Bangkok 10330, Thailand

E-mail address: sajee.p@chula.ac.th 


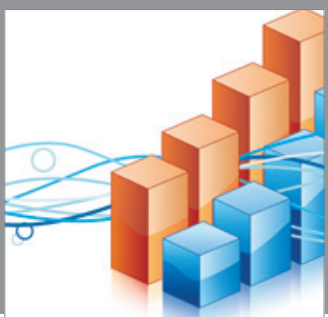

Advances in

Operations Research

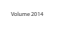

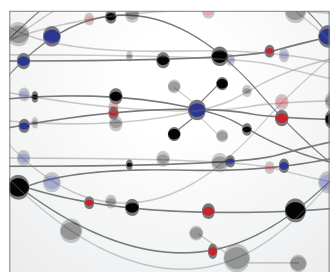

\section{The Scientific} World Journal
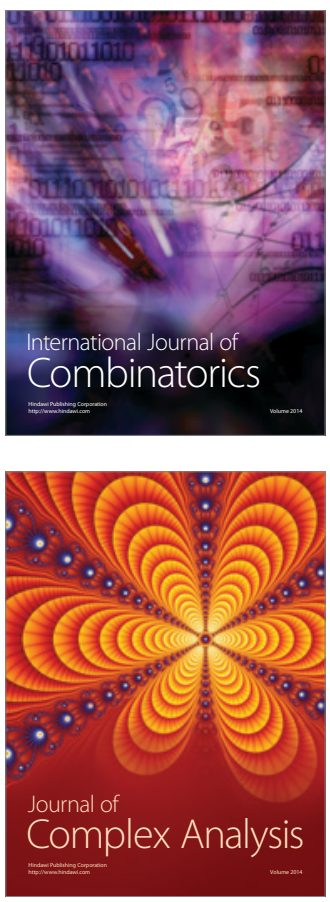

International Journal of

Mathematics and

Mathematical

Sciences
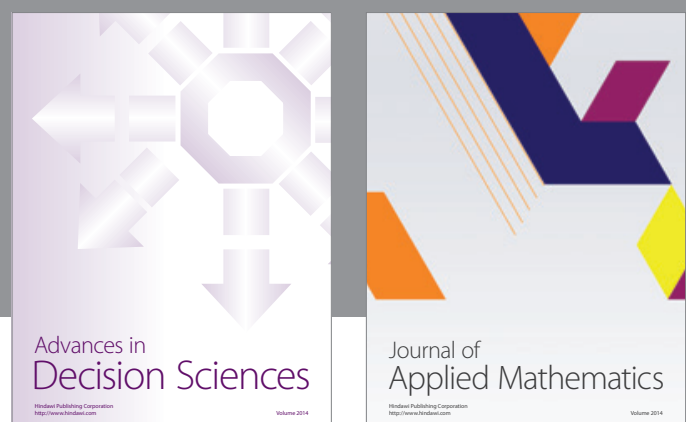

Journal of

Applied Mathematics
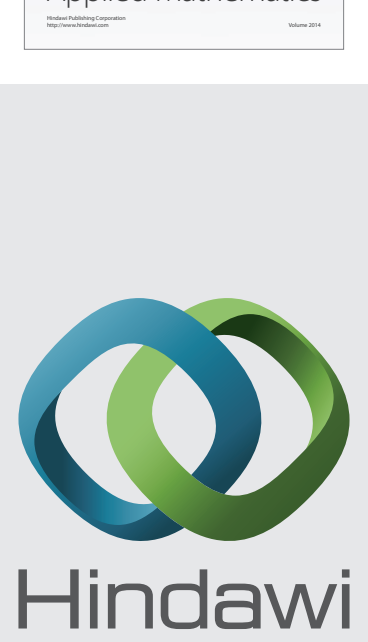

Submit your manuscripts at http://www.hindawi.com
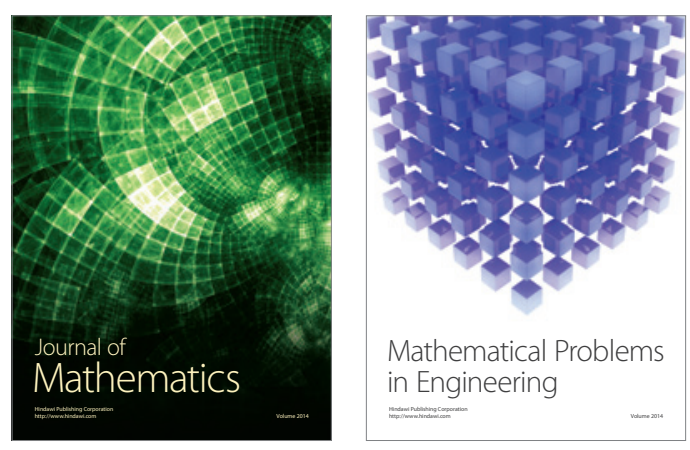

Mathematical Problems in Engineering
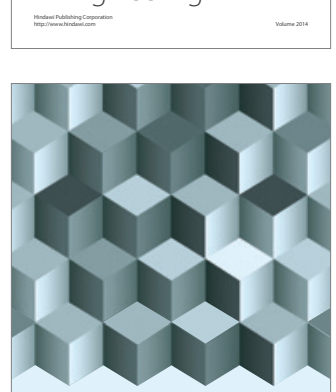

Journal of

Function Spaces
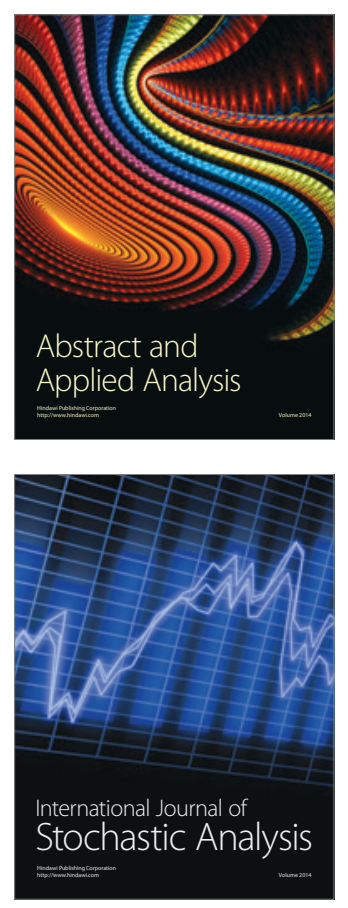

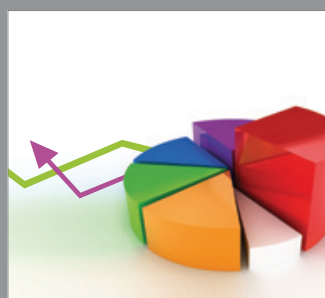

ournal of

Probability and Statistics

Promensencen
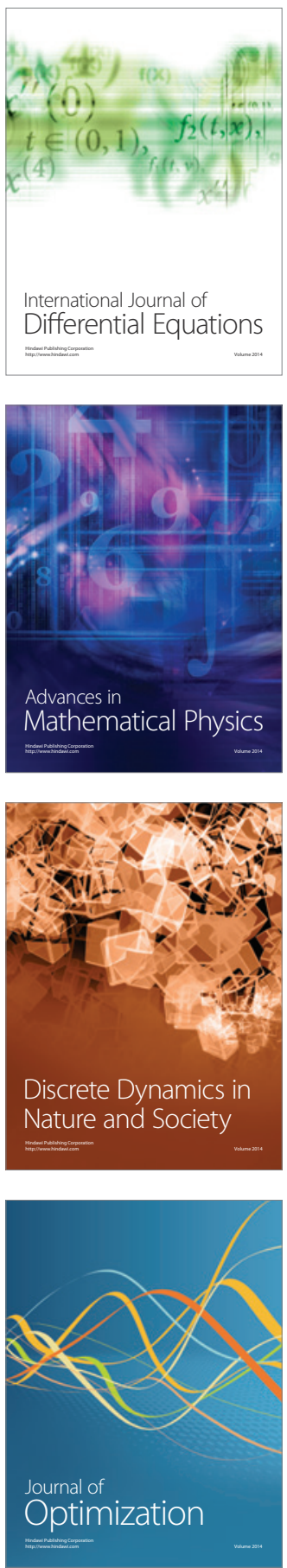